\title{
Correction to: PANLAR consensus statement on biosimilars
}

S. C. Kowalski ${ }^{1}$ (D) - J. A. Benavides ${ }^{2}$ • P. A. Beltrán ${ }^{2}$ • C. Galarza-Maldonado ${ }^{3,4}$ • C. V. Caballero-Uribe ${ }^{3,5} \cdot$ E. R. Soriano ${ }^{3}$. C. Pineda ${ }^{3}$ - V. F. Azevedo ${ }^{3}$ - G. Avila-Pedretti ${ }^{6}$ A. M. Babini ${ }^{7}$ - A. Cachafeiro-Vilar ${ }^{8}$ - M. Cifuentes-Alvarado ${ }^{9}$.

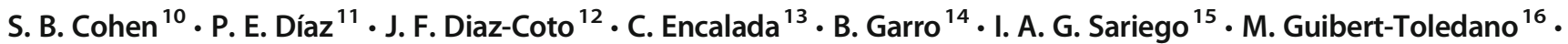
V. J. K. Rodriguez ${ }^{17}$ - M. E. L. Lopez ${ }^{17}$ - A. P. Ortega ${ }^{18}$ - A. S. Russell ${ }^{19} \cdot$ P. Santos-Moreno ${ }^{20} \cdot$ I. S. Terán ${ }^{21} \cdot$ A. Vargas $^{22}$. G. Vásquez ${ }^{20} \cdot$ R. M. Xavier ${ }^{23} \cdot$ D. X. Xibillé Firedman ${ }^{24} \cdot$ E. Mysler $^{7} \cdot$ J. Kay ${ }^{10}$

Published online: 20 May 2019

(C) International League of Associations for Rheumatology (ILAR) 2019

Correction to: Clinical Rheumatology (2019) 38:1497-1508

https://doi.org/10.1007/s10067-019-04496-3

The authors regret that the original version of this article, unfortunately, contained error. The two co-authors of the mentioned above article were incorrect. The authors should have been "P. A. Beltrán" instead of "P. A. B. Roa" and "J. F. Diaz-Coto" instead of "L. Diaz Soto". The correct authors are now presented correctly in this article.

The remainder of the article remains unchanged.

Publisher's note Springer Nature remains neutral with regard to jurisdictional claims in published maps and institutional affiliations.

The online version of the original article can be found at https://doi.org/ 10.1007/s10067-019-04496-3

S. C. Kowalski

sckowalskii@gmail.com

Extended author information available on the last page of the article 


\section{Affiliations}

S. C. Kowalski ${ }^{1}$ (I) J. A. Benavides ${ }^{2}$ •P. A. Beltrán ${ }^{2} \cdot$ C. Galarza-Maldonado ${ }^{3,4} \cdot$ C. V. Caballero-Uribe ${ }^{3,5} \cdot$ E. R. Soriano ${ }^{3}$. C. Pineda ${ }^{3}$ - V. F. Azevedo ${ }^{3}$ - G. Avila-Pedretti ${ }^{6}$ - A. M. Babini ${ }^{7}$ - A. Cachafeiro-Vilar ${ }^{8} \cdot$ M. Cifuentes-Alvarado $^{9}$.

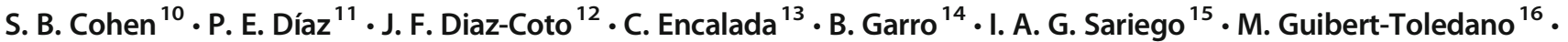
V. J. K. Rodriguez ${ }^{17} \cdot$ M. E. L. Lopez ${ }^{17} \cdot$ A. P. Ortega ${ }^{18} \cdot$ A. S. Russell ${ }^{19} \cdot$ P. Santos-Moreno ${ }^{20} \cdot$ I. S. Terán ${ }^{21} \cdot$ A. Vargas $^{22}$. $^{2}$ G. Vásquez ${ }^{20} \cdot$ R. M. Xavier ${ }^{23}$ - D. X. Xibillé Firedman ${ }^{24} \cdot$ E. Mysler $^{7} \cdot$ J. Kay ${ }^{10}$

1 Universidade Federal do Paraná, R. Gen. Carneiro, 181 - Alto da Glória, Curitiba, PR 80060-900, Brazil

2 Cencis, Av. Cra 45 \# 114-78 Edificio Espectrum, oficina 101, Bogota, Colombia

3 PANLAR (Pan American League of Associations for Rheumatology), 2200 Lake Boulevard NE, Atlanta, GA 30319, USA

4 UNERA-Unidad de Enfermedades Reumaticas y Autoinmunes, Corporacion Medica Monte Sinai, 010107 Cuenca, Ecuador

5 Hospital Universidad del Norte-Unidad de Reumatologia, Calle 30 Via Al Aeropuerto, Soledad-Atlantico, Colombia

6 Sociedad Paraguaya de Reumatología, Pitiantuta 613 esq. Juan de Salazar, Asuncion, Paraguay

7 Sociedad Argentina de Reumatología, Callao 384 Piso 2 Dto 6, CABA, C1022AAQ Buenos Aires, Argentina

8 Sociedad Panameña de Reumatología, Boulevard Pacífica y Vía Punta Darién, Panama City, Panamá

9 Asociación Guatemalteca de reumatología, 10a. Calle 6-40. Zona 9 Clínica 1.2do Nivel Edificio Salucentro, Guatemala City, Guatemala

10 American College of Rheumatology, 2200 Lake Boulevard NE, Atlanta, GA 303192200, USA

11 Sociedad Boliviana de Reumatología, Instituto Boliviano de Reumatología, Av. Irala, Santa Cruz de la Sierra, Bolivia

12 Asociacion Costarricense de Reumatologia, Reumatología, Hospital México, Caja Costarricense del Seguro Social, San Jose, Costa Rica
13 Sociedad Ecuatoriana de Reumatología, Edificio Torre Médica \#4 Piso 1, Coronel 2207 y cañar (994,96 km), Guayaquil, Ecuador

14 Sociedad Peruana de Reumatología, Av. José Pardo 138 oficina 1206 Miraflores, Lima, Peru

15 Sociedad Chilena de reumatología, Av. Nueva Providencia, 2214 Providencia, Región Metropolitana, Chile

16 Sociedad Cubana de Reumatología, Calzada de 10 de Octubre \#122, esquina Agua dulce Cerro, Havana, Cuba

17 Sociedad Dominicana de Reumatología, Primer Nivel Local 47 A. Diamond Mall, Santo Domingo, Dominican Republic

18 Asociación de Reumatología Nicaragüense, Bello Horizonte rotonda 4 1⁄2 cuadras al sur. J II 9, Managua, Nicaragua

19 Canadian Rheumatology Association, 9-6975 Meadowvale Town Centre Circle Suite 108 Mississauga, Toronto, Ontario L5N 2V7, Canada

20 Asociación Colombiana de Reumatología, Calle 94 \# 15-32 Oficina 603, Bogota, Colombia

21 Sociedad Venezolana de Reumatología, Apartado 5495, Caracas 1010, Venezuela

22 Sociedad Uruguaya de Reumatología, Av.Italia s/n esq. Las Heras 1er. Piso Sector, Montevideo, Uruguay

23 Sociedade Brasileira de Reumatología, Av. Brigadeiro Luís Antônio, 2466 - Jardim Paulista, Sao Paulo, SP 01402-000, Brazil

24 Colegio Mexicano de Reumatología, Paseo del Río No. 157 Col. Barrio Oxtopulco Universidad, Delegación Coyoacán, Cd., CP 04318 Mexico City, Mexico 